\title{
The Effect of Thermal Shocks on Wear of Exchangeable Sintered Carbide Inserts during the Cutting Process
}

Jana Petru, Tomas Zlamal, Ivan Mrkvica, Robert Cep

Faculty of Mechanical Engineering, VŠB - Technical University of Ostrava. 17. Listopadu 15/2172, Ostrava. Czech Republic. E-mail: jana.petru@vsb.cz, tomas.zlamal@vsb.cz, ivan.mrkvica@vsb.cz, robert.cep@vsb.cz

The article deals with testing of exchangeable cutting inserts from sintered carbide and determining of suitable chemical composition for their production. The experimental part was based on evaluation of cutting edge insert resistance against heat waves and thermal shocks during the milling process of commonly used stainless steel 1.4301 (X5CrNi18-10). It was determined amount, types and measured lengths each cracks occurred because of heat waves during the milling process for evaluation of proper chemical composition, grain size and content of cobalt for their production.

Keywords: sintered carbide insert, cutting, stainless steels, thermal shocks, heat cracks

\section{Acknowledgement}

Article has been done in connection with project Students Grant Competition SP 2014/105 financed by the Ministry of Education, Youth and Sports and Faculty of Mechanical Engineering VŠB-TUO.

\section{References}

[1] STAHL, J-E. METAL CUTTING (2012) Theories and models. Sweden: Division of Production and Materials Engineering. Lund University Sweden, p. 580. ISBN 978-91-637-1336-1.

[2] KOUŘIL, K. Odolnost slinutých karbidů proti teplotním a mechanickým rázům. [Online]. 2004 [cit. 14.3.2014]. Available from: http://www.mmspektrum.com/clanek/odolnost-slinutych-karbidu-proti-teplotnim-a-mechanickym-razum.html.

[3] HUMÁR, A. (2008) Materiály pro řezné nástroje. Praha: MM publishing, s. r. o., 235 s. ISBN 978-80-254-2250-2.

[4] MASTUDA, T., KIMURA, T., MASTUBARA, H. (2012) Evaluation Method of Thermal Shock Resistance of Cermets. Journal of the Japan Society of Powder and Powder Metallurgy, vol 59, no. 11, pp. 637-644. ISSN 0532-8799.

[5] ČEP, R., JANÁSEK, A., ČEPOVÁ, L., PETRU゚, J., HLAVATÝ, I., CAR, Z., HATALA, M. (2013) Experimental testing of exchangeable cutting inserts cutting ability. Tehnicki Vjesnik, vol. 20, Issue 1, pp. 21-26. ISSN 1330-3651.

[6] FERNÁNDES-ABIA, A. I., BARREIRO, J., et. al, (2013) Behaviour of PVD coatings in the turning of austenitic stainless steels. Manufacturing Engineering Society International Conference, MESIC 2013; Zaragoza; Spain; $26-28$ June 2013, pp. 133-141. ISSN 1877-7058.

[7] SECO CZ. Příručka pro technology - Opotřebeni se nevyhneme! [Online]. 2012 [cit. 14.3.2014]. MM : Trendy / Obrábění, p. 56. Available from http://www.mmspektrum.com/clanek/prirucka-pro-technology-opotrebeni-se-nevyhneme.html

[8] Kovosvit M.A.S. MCV 1270, [online], Available from: http://www.masmachinetools.com/en/machine/mcv-1270

[9] MRKVICA, I., NESLUŠAN, M., KONDERLA, R., JURKO, J., PANDA, A. (2013) Cutting forces by turning of Inconel 718 with insert from different materials. Manufacturing Technology, vol. 13, no. 4. pp. 499-504. ISSN 1213-2489.

[10] ISO 3685:1993 (1993). Tool-life testing with single-point turning tools, Geneva, International Organization for Standardization, $48 \mathrm{p}$.

[11] STANČEKOVÁ, D., ŠEMCER, J., DERBAS, M., KURŇAVA, T. (2013) Methods of measuring of residual stresses and evaluation of residual state of functional surfaces by x-ray diffractometric methods, Manufacturing technology, vol. 13, no. 4, pp. 547-552. ISSN 1213-2489.

[12] CEP, R., JANÁSEK, A., PETRŮ, J. ČEPOVÁ, L. CZAN, A., VALÍČEK, J. (2013) Hard machinable machining of cobaltbased superalloy. Manufacturing technology, vol. 13, no. 2, pp. 1421-147. ISSN 1213-2489. 